\title{
ELEMENTOS PARA LA DEFINICIÓN TERRITORIAL DEL YACIMIENTO PREHISTÓRICO DE VALENCIA DE LA CONCEPCIÓN (SEVILLA)
}

\section{ELEMENTS FOR THE TERRITORIAL DEFINITION OF THE PREHISTORIC SITE OF VALENCINA DE LA CONCEPCIÓN (SEVILLA)}

\author{
por \\ JUAN MANUEL VARGAS JIMÉNEZ
}

RESUMEN Sobre la base de un análisis del medio físico de Valencina y su entorno, los referentes presentados para la definición del enclave prehistórico, se fundamentan en la sistematización de los diferentes elementos arqueológicos, en su implantación espacial, así como su relación con una topografía sustentadora que hemos tratado de restituir.

De ello resulta una propuesta de definición en la que partiendo de una probable área nuclear se distinguen de momento dos ámbitos principales, el de ocupación habitacional y productiva, así como el exclusivo de necrópolis, que valorados en su máxima extensión conjunta ocupan una superficie total de unas 468.80 hectáreas.

ABSTRACT . On the base of an physical means analysis of Valencina and their environment, the references
presented for the definition of the prehistoric place they are based in the systematizing of the
different archaeological elements in their space installation, as well as their relationship with
a supportive topography that we have tried to restore.
Of it finds a definition proposal in which leaving of a probable nuclear area is distinguished
at the moment two main environments, that of residence and productive occupation, and the
exclusive of necropolis, that valued in their maximum combined extension they occupy a total
surface of some 468.80 hectares.

Palabras claves Valencina de la Concepción, Yacimiento Prehistórico, Medio Físico, Territorio, Áreas, Intervenciones, Elementos Arqueológicos.

Key words

Valencina of the Concepción, Prehistoric Site, Environment, Territory, Areas, Excavations, Archaeological Elements. 
En las elevaciones del Aljarafe, al oeste de Sevilla, se emplaza el yacimiento de época prehistórica de Valencina de la Concepción; ocupa en su mayor parte terrenos de esta localidad, aunque con extensiones hacia oriente, donde se adentra en el término de Castilleja de Guzmán.

La evolución de esta zona de alta relevancia histórica, no solo dentro del espacio referido al Aljarafe, sino en el conjunto del Bajo Guadalquivir, ha preservado vestigios que plantean una secuencia encuadrable en un Calcolítico - Bronce Antiguo.

Su significación se señala por las estructuras conservadas, ya sean los magníficos monumentos funerarios, o las no menos importantes construcciones vinculadas a la vida cotidiana de sus moradores (cabañas, silos, fosos, etc.), por la cantidad y calidad de los objetos y útiles asociados, y también por la importante dimensión que en este contexto adquiere el paisaje, en buena medida preservado, en el que se integran el conjunto de actividades.

A causa de estas variables y de los elementos de riesgo surgidos de la presión urbanística a la que se encuentra sometido el yacimiento, se puso en marcha mediante la colaboración entre el Ayuntamiento de Valencina y la Consejería de Cultura, la redacción de la Carta Arqueológica Municipal como documento de evaluación de la información arqueológica de Valencina y su entorno, en su relación con el medio que la ha sustentado y los factores de deterioro latentes. Con ello se persigue establecer unos parámetros de protección y conservación que posibiliten un desarrollo de la localidad equilibrado, una utilización racional del territorio y una gestión responsable de los recursos (Vargas 2004: 9).

En este contexto se subraya la necesaria definición territorial del yacimiento y sus diferentes hitos y/o sectores, entendida como el resultado de un diálogo histórico entre la caracterización física del espacio en su paulatina evolución y las evidencias conservadas de los individuos que se asentaron en él.

\section{ESPACIO}

Es preciso para comprender la morfología del propio yacimiento de Valencina y su significación en época prehistórica y la de los enclaves del entorno, establecer el marco físico en el que se desenvolvieron las actividades de sus habitantes. Se trata por tanto del análisis de aquellos aspectos que inciden en la ocupación del espacio, es decir en la relación hombre-medio, y para ello debemos partir de una aproximación a la situación actual que a la par es heredera de pretéritas condiciones paleoambientales.

Valencina y su entorno presentan desde el punto de vista del relieve, varias unidades diferenciables que de norte a sur serían: la campiña alomada de la cuenca del arroyo Pie de Palo, la vega del Guadalquivir, el escarpe del Aljarafe y finalmente, la plataforma elevada del Aljarafe donde se emplaza la actual población y se concentran los vestigios arqueológicos que constatan la ocupación de estas tierras desde época prehistórica.

La Campiña se corresponde con aquellos terrenos situados al norte, que forman parte de una amplia unidad comprendida entre las estribaciones de Sierra Morena, el pie de la cornisa septentrional del Aljarafe, el río Guadiamar al oeste y las riveras del Guadalquivir y del Ribera de Huelva al este (Campo de Gerena). De morfología llana presenta suaves ondulaciones y en nuestro sector, esta recorrida por algunos arroyos como el Pie de Palo y otros cauces de índole menor que desembocan en el Ribera de Huelva. Presenta suelos mayoritariamente de secano con problemas puntuales de drenaje y encharcamiento en las franjas asociadas a cauces. Las formaciones que acompañan a la mayoritaria presencia de laboreo agrícola, se reducen a herbazales y matorral junto a los cursos de agua.

La limítrofe Vega del Guadalquivir, por la propia caracterización de estos terrenos modelados por la acción niveladora de las láminas de inundación, proporciona un excelente soporte de cultivos, tanto de secano como de regadío; si bien la progresiva ocupación antrópica determina el retroceso de estos usos agrícolas. 
El Escarpe conforma una ladera de tendencia semicircular que ciñe la plataforma y pone en contacto las tierras altas con las inferiores de la campiña y la vega. En su coronación se distinguen distintas elevaciones mas o menos aplanadas, a partir de las que se marcan desniveles de entre el 20 y el $30 \%$. Los suelos son inadecuados para el cultivo, presentando drenajes excesivos e importantes problemas de erosión. Predominan las formaciones de matorral y herbazales, con especies freatófitas como zarzas y cañas que aprovechan la afloración de aguas subterráneas que se producen en la zona de contacto entre las formaciones acuíferas de la unidad del Aljarafe y las margas subyacentes.

Finalmente la Plataforma del Aljarafe, ligeramente inclinada hacia el suroeste arranca en las márgenes del río Pudio aproximadamente a unos $100 \mathrm{~m}$ de cota, alcanzando su máxima altitud en el Cerro de la Cruz con una cota cercana a los $165 \mathrm{~m}$. Los suelos son parcialmente aptos para el laboreo, pues existen algunas limitaciones que no llegan a restringir la elección de cultivos; actualmente abundan las plantaciones de olivar, si bien se tiene constancia como a finales del siglo XIX y buena parte del XX existían viñedos en distintos ámbitos de esta plataforma. En determinadas áreas el abandono del laboreo con el arranque del olivar ha provocado la formación de herbazales y zonas improductivas con notables problemas de erosión y la probable intencionalidad de generar expectativas urbanísticas.

En su conjunto el material geológico de base presenta un origen terciario, a excepción de los rellenos cuaternarios documentados en los terrenos bajos correspondientes a la llanura aluvial.

En el sector de campiña se disponen las margas grisáceas del Mio-Plioceno. Litológicamente se trata de margas arcillosas o carbonatadas y en algunos casos ligeramente limosas, con pasadas de yeso y óxidos de hierro que cabría definir desde el punto de vista granulométrico como arcillas limosas o limos arcillosos.

Sobre ellas y ocupando el reborde del Aljarafe aparece un tramo de alternancia de limos y margas con algunas intercalaciones de arenas y areniscas correspondientes al Plioceno Inferior. Se ha detectado la presencia de óxido de hierro, observándose también como entre cada tramo arcilloso y el limo arenoso existe una etapa de interrupción sedimentaria marcada por la presencia de huellas de bioturbación, que en la mayoría de los casos queda truncada por el paquete arenoso. Del análisis granulométrico se deduce que se trata de arcillas-limo-arenosas.

Finalmente y ocupando el tramo sedimentario superior de la plataforma se localizan limos arenosos de tonalidad amarillenta también del Plioceno Inferior. La litología es de limos y arenas con algunos niveles de areniscas intercalados en los niveles más altos. También se ha observado la presencia de óxidos de hierro y respecto de la granulometría se definen como arenas y limos arcilloso-arenosos (Galán y Pérez 1989: 38-57). Se corresponde éste con el paquete en el que se excavan el conjunto de estructuras arqueológicas individualizadas en el yacimiento, con algunos casos de presencia sectorial de una cubierta arcillosa de tonalidad rojiza que cierra la secuencia.

En síntesis podemos establecer como desde el punto de vista del relieve e incluso de la sedimentología, dentro del territorio de Valencina y su entorno próximo, se pueden distinguir dos áreas principales, por un lado los terrenos bajos fuertemente influenciados por la dinámica fluvial, y por otro la corona superior correspondiente a las elevaciones del Aljarafe.

Sin duda las transformaciones históricas mas notables cabe relacionarlas con los terrenos bajos, al pie de la plataforma, donde se sitúa la llanura aluvial del Guadalquivir que comprende una franja de entre 6 y $7 \mathrm{~km}$ de anchura al este de Valencina, la cual se ve surcada por un gran canal divagante-el Guadalquiviry sus tributarios, así como meandros actuales y paleocauces.

En la reconstrucción general de la secuencia de la llanura, se arranca de un sustrato de margas del MioPlioceno correspondientes al episodio final del relleno marino de la depresión del Guadalquivir que presenta una morfología oblicua tanto hacia oriente como occidente en cuyos extremos aflora. Sobre él y mediante contacto erosivo se disponen varios metros de gravas y escasas arenas correspondientes al sistema de terrazas fluviales del Guadalquivir que presentan un techo de morfología fuertemente ondulada y ascendente de oeste a este. En el sector concreto en torno al área urbana de Sevilla, se han identificado tres niveles de 
terrazas; el superior (T1) se desarrolla en cotas de 40-50 m, apareciendo únicamente al nordeste; la terraza media (T2) también al nordeste, ocupa cotas entre 20 y $39 \mathrm{~m}$., siendo datada por la presencia de Elephas antiquus en el Pleistoceno medio superior; y la inferior (T3) sobre la que encaja el cauce del Guadalquivir presenta cotas variables entre 10 y 15 m (Galán y Pérez 1989: 40 y 102). Finalmente sobre esta última se disponen los materiales recientes de origen aluvial conformantes de la llanura propiamente dicha.

La configuración de este medio y su paulatina evolución se verá condicionada por el avance de las aguas marinas -transgresión flandriense-cuya culminación hacia el 4.000 a.n.e. aproximadamente, provocará la formación de una línea de litoral jalonada por cabos y golfos cuyas playas se situaban en tierras hoy interiores, sería este el caso del espacio actual de las Marismas del Guadalquivir, conformado como un verdadero golfo marino con su límite norte en el escarpe del Aljarafe y hacia levante los antiguos acantilados de Lebrija y Trebujena (Díaz del Olmo 1989: 19, Arteaga y Roos 1995: 329-339).

De este modo debemos considerar como primitivamente, la desembocadura del Guadalquivir se hallaba bastante más arriba que la actual, conforme a las reconstrucciones propuestas en las proximidades de Coria del Río, vertiendo a un golfo marino conocido en la antigüedad con el nombre de sinus Tartessii. Más arriba, el estuario prolongaba el ambiente litoral hasta la propia Sevilla, que entonces debía ser una especie de isla en medio de una vasta llanura aluvial. En época romana, el río anteriormente denominado Tartessos cambió su nombre por el de Baetis, ubicándose su desembocadura más abajo, a la altura de Lebrija, la acumulación sedimentaria habría reducido el antiguo golfo, convertido ahora en laguna litoral, el lacus Ligustinus de los romanos (Belén 2000: 79-80).

Este proceso de continentalización de las antiguas bahías y golfos se realiza tanto por el aumento de los aportes, y por tanto del espesor de los rellenos, como por un descenso del nivel marino hasta el nivel actual, es decir unos dos metros; de este modo se configuran abundantes sistemas de playas e islas-barreras que paulatinamente taponarán las grandes bahías y la desembocadura de los cursos fluviales. Siendo los actuales paisajes marismeños reflejo de esa progresiva colmatación que aún sigue abierta.

Sin embargo y a pesar de que dentro de este marco evolutivo, cabría precisar y puntualizar el comportamiento especifico de cada sector en concreto, parece que para época prehistórica la situación en el entorno de Valencina estaría marcada por la proximidad de ese importante golfo marino, y la presencia en las tierras bajas de una gran arteria fluvial que dada la cercanía de su desembocadura debía mostrar un ambiente de fuerte presencia hidrológica tanto en lo referido a la multiplicidad y/o amplitud de los cauces como en la extensión de las zonas inmediatas directamente influidas por su dinámica, generándose además, en este ambiente de estuario, unas condiciones poco saludables derivadas de la más que probable presencia de insectos que como ocurría hasta no hace mucho tiempo en el actual paraje de Doñana, supondrían una verdadera plaga.

Esta situación ha tenido y tiene su reflejo en la disposición y evolución de los cauces y humedales en el sector de Sevilla, con la profusión de cursos secundarios (Tagarete, Tamarguillo), paleocauces, islas (Isla Quijano, Isla de la Cartuja) y zonas inundables que se han podido reconocer por la toponimia (Prado de San Sebastián, Prado de Santa Justa), las investigaciones arqueológicas (Martín-Consuegra y otros 1998: 530-531, Romo 1999: 425-430) o el análisis de diferentes cartografías históricas (Córtés y otros 1992).

Las condiciones de habitabilidad eran poco favorables en estos terrenos bajos, lo que de alguna manera también se infiere si tenemos en cuenta las localizaciones arqueológicas prehistóricas, que en lo conocido hasta la fecha, se restringen junto a la propia Valencina y El Carambolo, a las evidencias reconocidas en la Univ. Laboral de Sevilla (Fernández y Alonso 1985: 9-26) o en el Cortijo de Miraflores (Jiménez 1999) que se sitúan justo en el límite de la llanura aluvial, a salvo del nivel máximo que alcanzaron las más importantes crecidas del río -como la del año 1709 o 1892 (Vanney 1970) o la más recientemente de 1963 (Baena y Díaz del Olmo 1997: 65-89)-, ambos en el borde de la terraza media (T2), en una cota homogénea situada en torno a los $+20 \mathrm{~m}$ y finalmente, al pie de dos cursos, el Guadaíra y el Miraflores, que desde el este entran en contacto con la llanura aluvial, precisamente en los puntos de ubicación de estos dos últimos enclaves arqueológicos (fig. 1). 


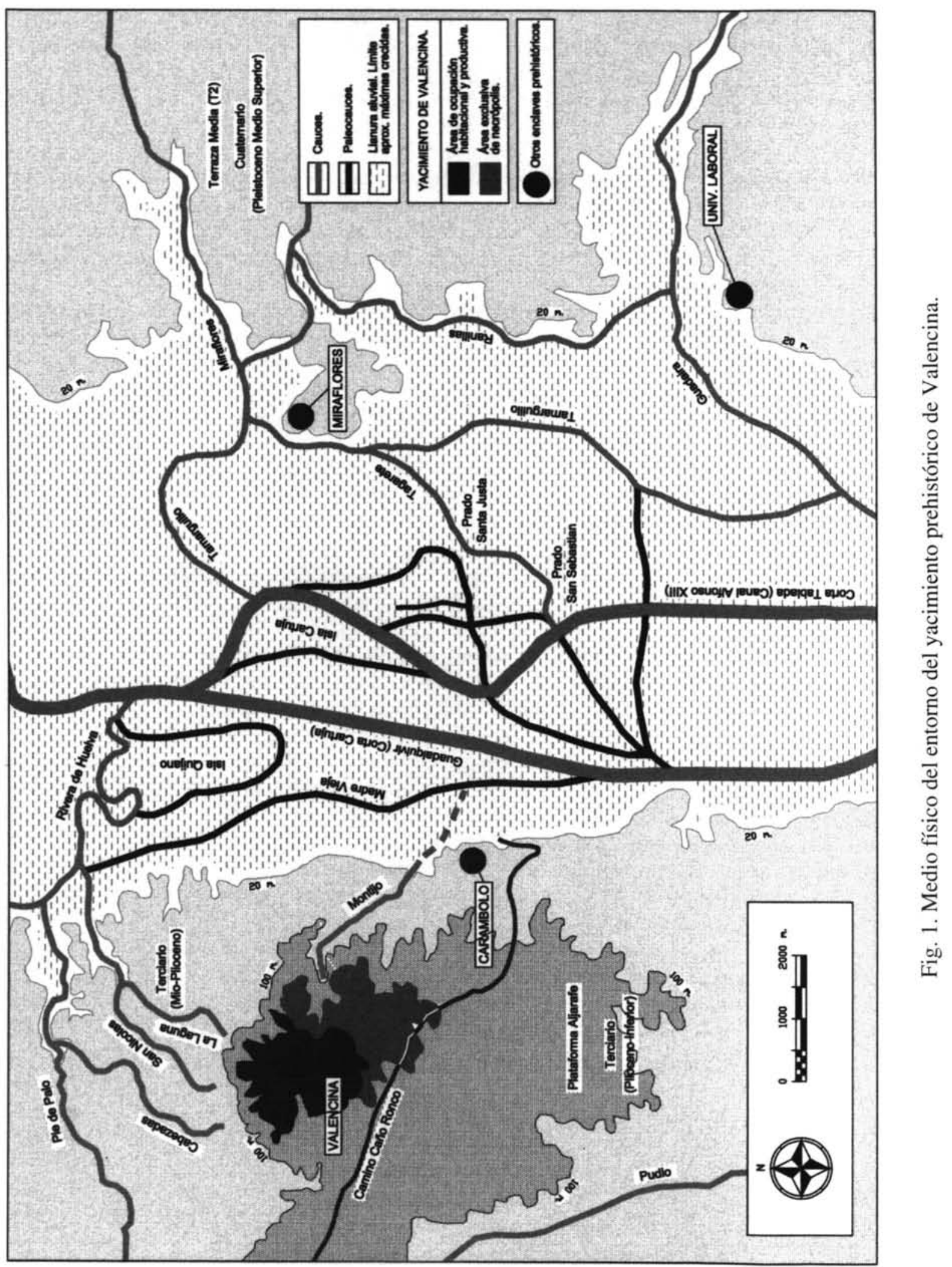


La paulatina colmatación del primitivo golfo marino y el avance de la desembocadura del Guadalquivir ya mencionadas, se manifiesta cauce arriba en una progresiva desecación y estabilización del medio, periódicamente alterada por inundaciones y riadas, como las numerosas recogidas para la edad moderna y contemporánea (Palomo 1878) que en su máxima implantación pueden ayudarnos a comprender primitivas fisonomías.

De este modo se justificaría la posición estratégica del yacimiento de Valencina ocupando las máximas altitudes del Aljarafe, suficientemente separado de las insalubres condiciones de las tierras bajas y de los inestables terrenos del escarpe, en un punto que además constituye el extremo oriental de la vía natural que pone en contacto los yacimientos mineros de Aznalcollar y la vía fluvial del Guadalquivir, y que posiblemente se perpetúe en un trazado equiparable al del actual camino de Caño Ronco.

Efectivamente en este extremo de la plataforma del Aljarafe, con material geológico de origen terciario, unas características sedimentológicas y un relieve actual que ya se han expuesto, se emplaza el yacimiento prehistórico. A pesar de que en este lugar las trasformaciones han sido de menor envergadura, cabe realizar algunas observaciones que nos ayudarán a entender mejor la disposición del enclave y su articulación interna. Para ello hemos tratado de reconstruir la primitiva topografía y los cauces relacionados como elementos condicionantes de la ocupación espacial a nivel micro. En base a un exhaustivo estudio de cotas, a la revisión de antiguas cartografías, así como al análisis de diversa información sobre pretéritas remociones y colmataciones sectoriales, ya fueran de origen natural o antrópico, pudimos reconocer seccionamientos de elevaciones y consiguientes rellenos de vertientes, cauces estacionales hoy ocultos, o sectores tradicionalmente sometidos a embalsamiento puntual.

Se constata así un proceso general de nivelación que erosiona las cumbres y rellena las depresiones, en una evolución que parte de la paulatina desmantelación de la vegetación autóctona, prosigue con la mayoritaria puesta en labor de los campos y la mecanización de las explotaciones; para culminar en época reciente con la trasformación de los suelos rústicos en urbanos. Por ejemplo tenemos constancia de recientes pérdidas de potencia estratigráfica de en torno al metro en la primitiva finca la Horca, o de una evolución del túmulo de La Pastora que muestra una considerable merma desde los dos metros sobre la cámara, constatados en el momento de su descubrimiento, a finales del siglo XIX.

Así, en la restitución realizada se observa una meseta superior central de forma tendente a cuadrangular y suaves laderas perimetrales, que se encuentra ceñida entre las líneas de cota 150 y 154 m alcanzándose valores máximos de $160 \mathrm{~m}$ de altitud en la mitad oriental; grosso modo coincidiría con los antiguos terrenos de La Estacada Larga y La Candelera. En torno a ella, rodeándola se dispone una morfología caracterizada por la presencia de pequeñas lomas o cerros enmarcados por cañadas más o menos acentuadas según se trate de los fuertes desniveles que acompañan a las elevaciones septentrionales o los de menor grado apreciables en el extremo sur. Al norte se disponen las altitudes correspondientes a Las Coronas, Cerro de la Cruz, Cerro de la Cabeza y Cerro Mármol, mientras que al sur los cabezos alomados -de menor altitud-se sitúan en los puntos álgidos de sectores hoy conocidos como Sitio de Valencina, Algarrobillo, La Horca o El Cuervo, estando en este sector acompañados de zonas endorreicas y de escorrentía no ocupadas, y que en la actualidad presentan fuertes rellenos de colmatación que enmascaran relieves anteriores más acentuados, si bien como pervivencia de ellos y hasta mediados del siglo XX eran sectores por los que puntualmente corría el agua (fig. 2).

Completaría la percepción de este paisaje una probable presencia de formaciones más o menos densas de bosque mediterráneo, de encinas, acebuches, etc. de la que resulta ilustrativa la propia etimología propuesta para el nombre de Valencina (Valle de la Encina), las referencias de antiguos catastros que señalan la presencia de esta especie arbórea hoy prácticamente desaparecida, así como también las analíticas de restos faunísticos prehistóricos que determinan la presencia de especies como el ciervo, el uro, el jabalí o el conejo, representativas del ecosistema circundante. 


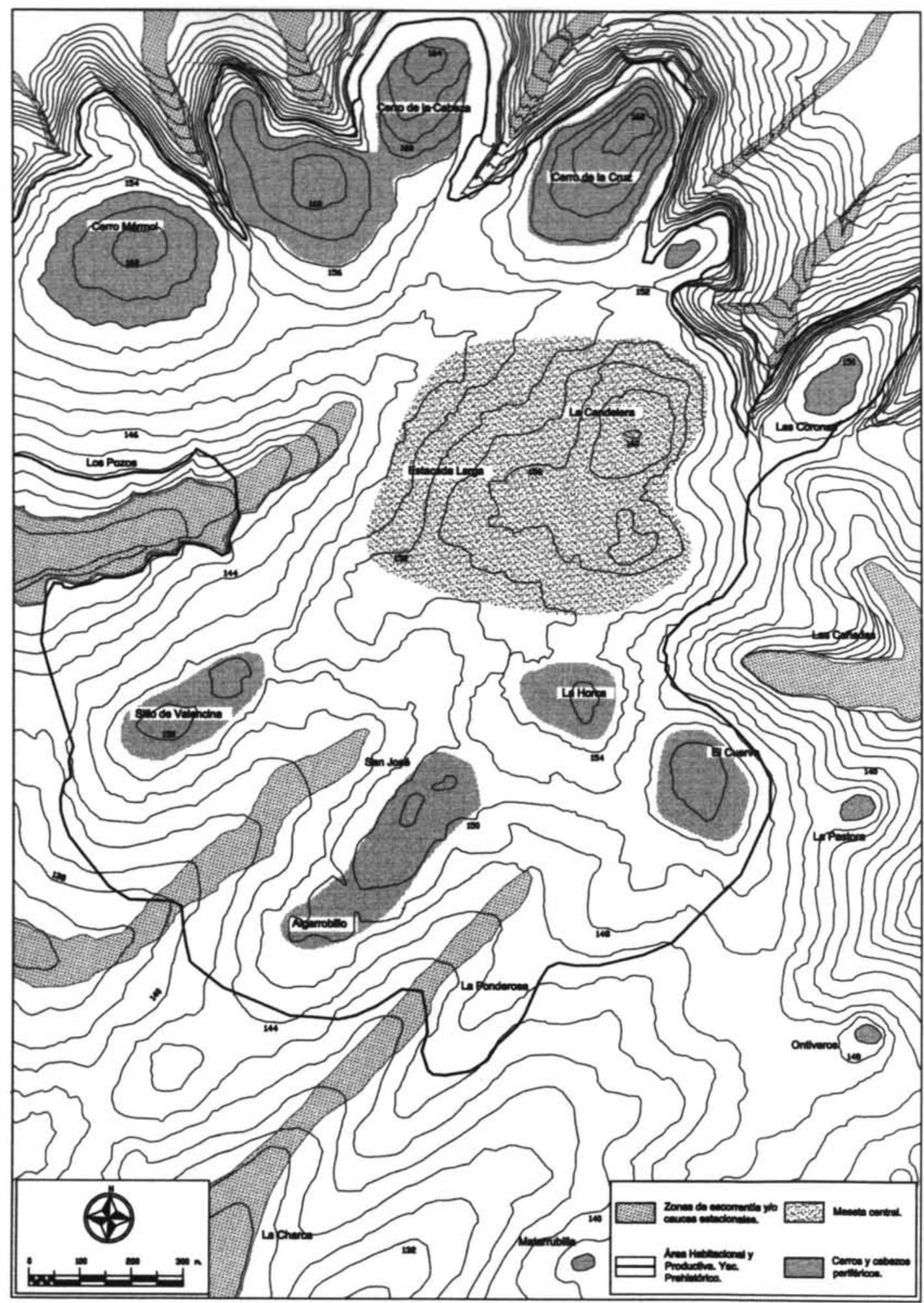

Fig. 2.- Restitución de la topografía antigua y cauces relacionados.

ISSN: 1133-4525 ISSN-e: 2255-3924

SPAL 12 (2003)

http://dx.doi.org/10.12795/spal.2003.i12.06 
Sin embargo, faltan a nuestro juicio más estudios de restos óseos que incrementando la muestra permitan conclusiones más exhaustivas, así como analíticas palinológicas que con la visión particularizada sobre la vegetación antigua, ofrezcan un enfoque complementario que posibilite certeras reconstrucciones paleoambientales.

\section{INDICADORES ARQUEOLÓGICOS}

Los elementos de base que articulan la vertebración territorial del yacimiento parten de los anteriores parámetros de carácter físico que condicionan en mayor o menor medida la ocupación antrópica. Junto a ellos y con los precedentes de las propuestas historiográficas, los indicadores utilizados para la definición espacial del enclave, se fundamentan en la revisión de las intervenciones arqueológicas realizadas hasta la fecha, para sistematizar la naturaleza de los diferentes hallazgos y su distribución espacial, en su interrelación con la evolución de la topografía sustentadora.

Las hipótesis mas recientes formuladas para la definición del ámbito ocupado por el yacim̃iento prehistórico de Valencina parten de un tronco común que establece una división entre dos ámbitos principales, el poblado y la necrópolis, a los que le acompaña en el último caso, la fijación de una zona de tránsito denominada área intermedia.

La primera propuesta corresponde al plano de la Delegación Provincial de la Consejería de Cultura de Sevilla, que en los años 90 y a instancias de J.M. Rodríguez e I. Santana (Santana 1993: 549), establece una amplia área para el poblado, extendida hasta el límite norte de la cornisa, por el sur ocupando las urbanizaciones más meridionales (Las Pilas y La Gloria) y por el oeste hasta el sector de la Hacienda de Torrijos. $\mathrm{Al}$ oriente y lindando con ella se dispondría la necrópolis que superando los límites del término municipal se adentra en Castilleja de Guzmán con un final coincidente con la línea de cota 100 m que marca el extremo de las elevaciones superiores de este borde de la cornisa (fig. $3 \mathrm{n}^{\circ} 1$ ).

En la siguiente referida al ámbito del poblado (Ruiz 1996 a: 41), se plantea una ocupación más restrictiva, con el límite norte por debajo de las cúspides de la plataforma, incluyendo la ladera meridional del Cerro Mármol y mayoritariamente centrada en el núcleo ocupado por la actual población. A poniente el borde de la delimitación lo marca la separación entre la urbanización Sitio de Valencina y la de Torrijos, con la inclusión más al norte de Villavalencina y la eliminación de la cuña dibujada por la Cañada de Los Pozos. El extremo sur se hace coincidir con el trazado del desvío de la carretera SE-510 (fig. $3 \mathbf{n}^{\circ} 2$ ).

Una variante de la anterior (Base de Datos Arqueos, I.A.P.H., 1996) dibuja un polígono con ocho vértices que dan cobertura a un área de poblado más o menos coincidente por el sur con los límites de la que antecede, con la particularidad de la inclusión en el extremo del perímetro septentrional, del Cerro de la Cruz y la ladera meridional del Cerro de la Cabeza o Cerro Barro (fig. $3 \mathrm{n}^{\circ} 3$ ).

Por último O. Arteaga y R. Cruz-Auñón plantean una hipótesis articulada conforme a una compartimentación tripartita de áreas de carácter funcional, en la que se diferencian el poblado en un sector central, al oriente del cual se dispone la llamada área intermedia definida por sus implicaciones de carácter económico y caracterizada por la abundancia de estructuras de almacenaje; y finalmente en el extremo sureste, la necrópolis (fig. $3 \mathrm{n}^{\circ} 4$ ). Los límites en conjunto de estas tres áreas, son más restrictivos, desechándose, esencialmente, todo el sector al occidente del casco antiguo de Valencina, y reduciéndose el ámbito de la necrópolis en su sus límites oriental y occidental (Arteaga y Cruz Auñón 1999 a: 590). 
(ㄱ)
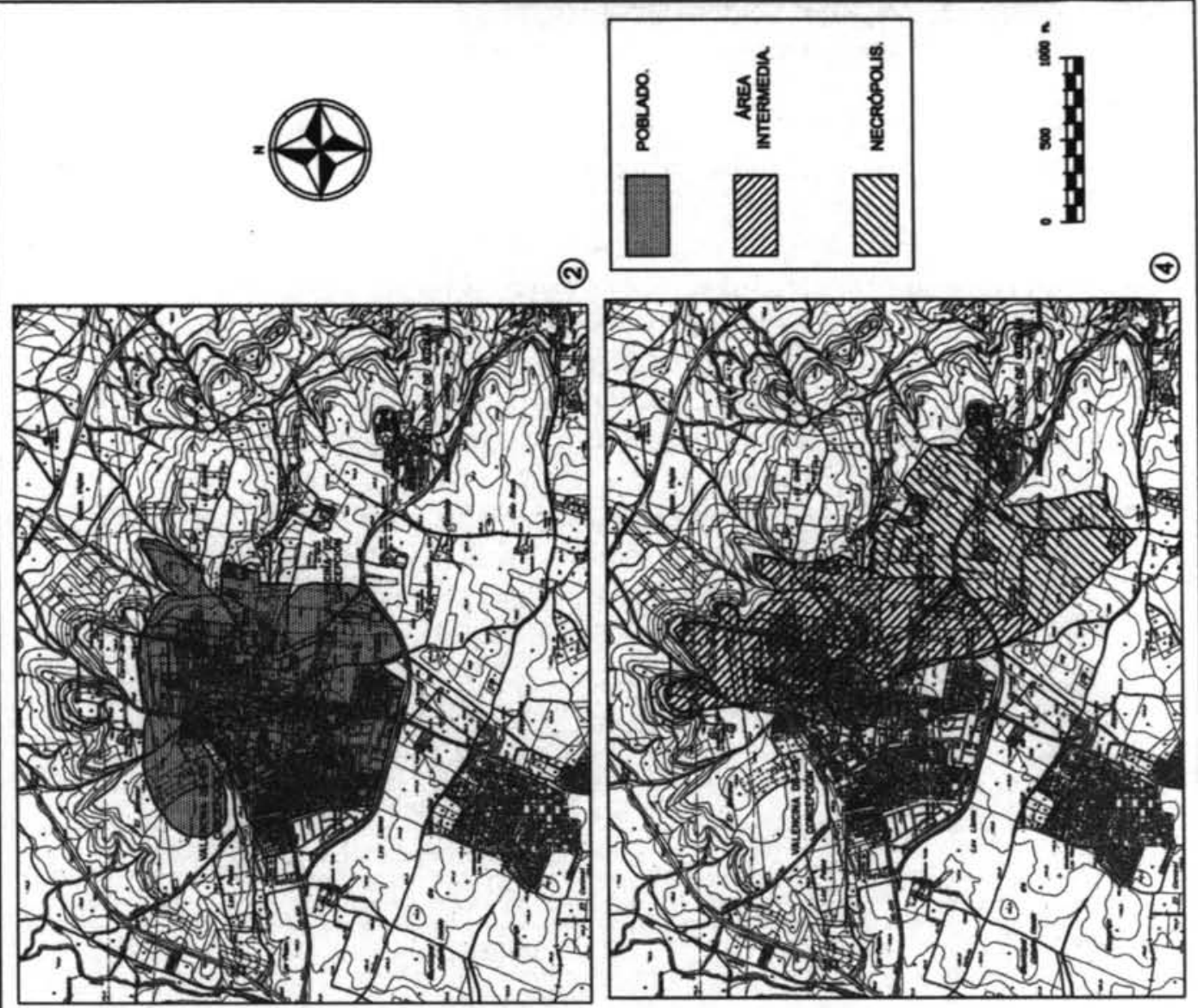

$\odot$
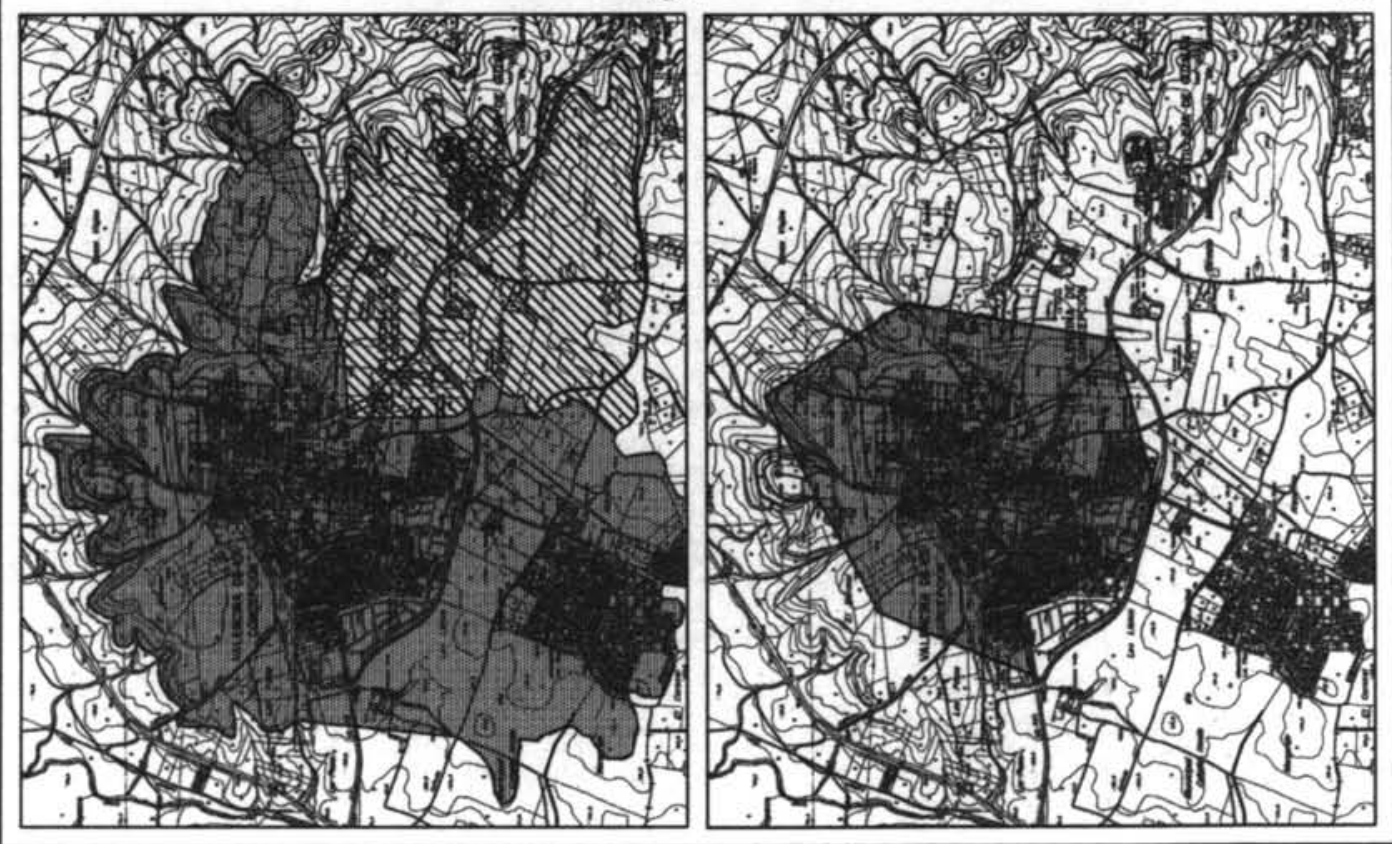

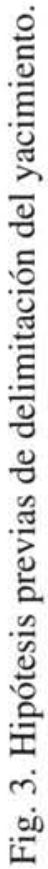


El conjunto de intervenciones arqueológicas que arrancan con el descubrimiento de La Pastora y la excavación de Tubino en 1868, constituyen el soporte indispensable para el análisis de las características del yacimiento y su diagnosis territorial. Su revisión y sistematización nos ha deparado la obtención de un amplio corpus informativo con más de 70 actuaciones, cuyo estudio nos ha planteado no pocas dificultades derivadas de los problemas de acceso a la información y los diferentes grados de plasmación de los datos extraídos, con algunos ejemplos de alta rentabilidad científica y otros realmente someros.

Los elementos arqueológicos individualizados en asociación a su ubicación diferencial, constituyen los primordiales indicadores de extensión y distinción zonal en el yacimiento prehistórico; de su análisis se han deducido otras particularidades que agrupadas van definiendo características homogéneas del yacimiento, como la naturaleza de la deposición estratigráfica (potencia, secuencia, etc.) o de los hallazgos estructurales (tipología, dimensiones, proyecciones, etc); los distintos grados de conservación del sustrato o las perspectivas en referencia a las potencialidades arqueológicas subyacentes.

\section{Síntesis Tipológica de los Hallazgos Prehistóricos según las Intervenciones Arqueológicas} de Valencina de la Concepción y Castilleja de Guzmán.

[Fuente: Carta Arqueológica Municipal (Vargas 2004)]

\begin{tabular}{|l|l|}
\hline \multicolumn{1}{|c|}{ LOCALIZACIÓN } & \multicolumn{1}{c|}{ TIPOLOGÍA } \\
\hline Dolmen de La Pastora. & Estructura Funeraria. \\
\hline Sepulcro de Caño Ronco & Estructura Funeraria. \\
\hline Dolmen de Matarrubilla. & Estructura Funeraria. \\
\hline Dolmen de Ontiveros. & Estructura Funeraria. \\
\hline La Perrera. Crtra. Santiponce. & Restos Área de Hábitat. \\
\hline Los Cabezuelos. & Estructura Funeraria. \\
\hline Cortes A,B,C y D. La Perrera. & Fondo Cabaña(?), Fosos, Silos. \\
\hline Tholos del Cerro de la Cabeza o Cerro Barro. & Estructura Funeraria. \\
\hline Sitio de Valencina. & Restos Área de Hábitat y Estructura Funeraria (?). \\
\hline Cerro de la Cabeza o Cerro Barro. & Fondo Cabaña, Fosos, Silos, Pozos. \\
\hline Cerro del Goro o Cerro Mármol. & Restos Área de Hábitat. \\
\hline C/ Blas Infante, 8, antigua Buen Alcalde, 11. & Restos Área de Hábitat. \\
\hline C/ Ebro. & Restos Área de Hábitat. \\
\hline C/ Duero, 32. Estaca Larga I. & Restos Área de Hábitat. \\
\hline C/ Ebro, 2. Estaca Larga II. & Restos Área de Hábitat. \\
\hline C/ Guadalquivir, 30 ant. 32. & Restos Área de Hábitat. \\
\hline C/ Cervantes, 7. & (?) \\
\hline C/ Genil. & Restos Área de Hábitat. \\
\hline C/ Itálica, 6. Guadalquivir prolongación. & Restos Área de Hábitat. \\
\hline C/ Tajo. & Restos Área de Hábitat. \\
\hline C/ Primero de Mayo antigua Veinticuatro de Julio. & Restos Área de Hábitat. \\
\hline C/ Duero, 41. & Restos Área de Hábitat. \\
\hline
\end{tabular}




\begin{tabular}{|c|c|}
\hline LOCALIZACIÓN & TIPOLOGÍA \\
\hline C/ Guadalquivir, 6. & Restos Área de Hábitat. \\
\hline C/ Guadalquivir, 24. & Fondo de Cabaña (?). \\
\hline C/ Félix Rodríguez de la Fuente. & Silos. \\
\hline C/ Guadalquivir, 28. Guadalquivir esquina Ebro. & Restos Área de Hábitat. \\
\hline Las Coronas, La Bajara. & Silos. \\
\hline Dolmen de Los Veinte. & Estructura Funeraria. \\
\hline Cerro de la Cruz. & Restos Área de Hábitat \\
\hline Pastora $2-3-4$ y 5 . & Estructuras Funerarias. \\
\hline La Quemá. & Restos Área de Hábitat (?). \\
\hline Polideportivo municipal. & Cabañas, Foso, Silo, Pozo. \\
\hline Roquetito. & Estructuras Funerarias. \\
\hline Finca Ntra. Sra. de los Reyes. & Estructura Funeraria. \\
\hline Finca de la Horca. & Foso, Silos. \\
\hline C/ Duero, 39. & Fondo Cabaña, Silos. \\
\hline C/ Duero, 4. & Restos Área de Hábitat. \\
\hline La Emisora / Finca La Candelera. & Fondos de Cabañas, Foso, Silos, Pozo. \\
\hline La Cima. & Fosos, Silos. \\
\hline Nuestra Señora de los Reyes. Ontiveros. & Estructura Funeraria. \\
\hline C. de la Cruz - Finca La Horca. Zanja Aljarafesa. & Restos Área de Hábitat (?). \\
\hline Antiguo depósito Castilleja de Guzmán & Estructura Funeraria. \\
\hline La Escalera. & Estructura Funeraria \\
\hline La Gallega. & Fondo de Cabaña (?), Silos. \\
\hline El Algarrobillo. & Fondo Cabaña, Silo, Estructura Funeraria. \\
\hline Centro Deportivo “Manuel Muñoz". & Estructuras Funerarias. \\
\hline Mataherrera. La Curva. Zanja Aljarafesa. SE-510. & Estructuras Funerarias. \\
\hline Urbanización El Mirador de Itálica. & Fondos de Cabañas, Fosos. \\
\hline El Cuervo. & Silos. \\
\hline La Estacada Larga. & Foso, Silos. \\
\hline C/ Mariana Pineda, 40E. Ctra. de Salteras. Pozoblanco. & Fondos de Cabañas, Silos, Foso. \\
\hline Divina Pastora. Urb. Señorío de Guzmán (Castilleja). & Estructuras Funerarias. \\
\hline La Alcazaba. Parcela MA - 4. & Foso, Silos. \\
\hline C/ Alamillo, C/ TVE. & Silos. \\
\hline $\mathrm{C} /$ Alamillo esquina a C/ TVE. & Silos, Foso, Fondo de Cabaña. \\
\hline $\begin{array}{l}\text { C/ Manuel Altolaguirre, Parc. } 8 \text { y 9. Antigua } \\
\text { C/ Mariana Pineda. Ctra de Salteras. }\end{array}$ & Ausencia de estructuras. \\
\hline C/ La Emisora, 3, 4 y 5. C/ TVE. & Silos. \\
\hline
\end{tabular}




\begin{tabular}{|l|l|}
\hline \multicolumn{1}{|c|}{ LOCALIZACIÓN } & \multicolumn{1}{c|}{ TIPOLOGÍA } \\
\hline Zona Norte de Castilleja. Montelirio. & Estructuras Funerarias. \\
\hline C/ TVE, 14A antigua 10 A. & Ausencia de estructuras. \\
\hline Pza. Ntra Sra. de la Estrella, 1. Ayuntamiento. & Ausencia de estructuras. \\
\hline Zona Norte de Castilleja. M-1. Plan Parcial 3. & Estructuras Funerarias. \\
\hline La Alcazaba. Na . Sra. de la Esperanza. Parcela MB -4. & Fondos de Cabañas, Zanja, Silos. \\
\hline C/ Mariana de Pineda esquina a Bulería. & Ausencia de estructuras. \\
\hline Plan Parcial Matarrubilla. & Silos, Foso. \\
\hline Zanja Cerro Cruz - C/ TVE. Centro emisor. & Silo (?), Fondo de cabaña. \\
\hline C/ Manuel Altolaguirre, Parcela 3. & Ausencia de estructuras. \\
\hline Parque público La Gallega. & Silo y Fondo de cabaña (?). \\
\hline C/ Manuel Altolaguirre, Parcela 11. & Fondo de cabaña (?). \\
\hline La Alcazaba. Parcelas B-2 y B-5. & Silos y estructuras de habitación. \\
\hline Cerro Mármol. La Herrerilla. & Estructura funeraria (?) \\
\hline C/ Alamillo. Tramo entre C/ Azucena y C/ Fray Ambrosio. & Silos (?) \\
\hline Camino de los Mariscales. & Silo. \\
\hline
\end{tabular}

\section{PROPUESTA DE DEFINICIÓN}

La articulación de los anteriores elementos en su conjunción con el marco espacial establecido, permiten ir definiendo parámetros territoriales de ocupación y extensión del yacimiento prehistórico. Sin embargo es necesario precisar que en cualquier caso se trata de un proceso abierto en el que la sistematización e incremento de las investigaciones, no solo irá precisando los límites máximos fijados sino que también determinará la individualización de diferentes sectores dentro del conjunto delimitado. Igualmente la percepción de las diferentes fases cronológicas de la evolución del enclave y su paulatina plasmación espacial conllevará el establecimiento de diversos ámbitos en sincronía clarificadores de una realidad arqueológica que se nos antoja bastante más compleja y heterogénea de lo que en apariencia pudiera parecer.

La distribución de los diversos elementos arqueológicos documentados en diferentes intervenciones presentan un panorama en el que lo primero que se evidencia es la separación entre aquellos situados en el extremo oriental, de naturaleza funeraria y el resto, en el que predominan los que denominamos habitacionales o productivos (cabañas, silos, etc.). Estos últimos se extienden desde los límites más septentrionales de la cornisa (Cerro de la Cabeza) hasta el sur, donde en El Algarrobillo entran en contacto con estructuras funerarias. Por el oeste el Cerro del Mármol, las localizaciones estructurales de la intervención en C/Mariana Pineda, 40 (Ruiz 1996 b) y las evidencias negativas apreciables en algunas parcelas de la urbanización Torrijos (por ejemplo C/ M. de Pineda esquina a Bulería), vienen a establecer un límite por este sector, seccionado en forma de cuña hacia el interior por la depresión natural que supone la Cañada de Los Pozos. Finalmente en el extremo oriental los hallazgos acaecidos en la Urbanización El Mirador de Itálica (Ruiz 1994: 511 - 516), las evidencias de Las Coronas/La Bajara y los resultados de la intervención arqueológica de El Cuervo (Arteaga y Cruz Auñón 1999 b: 608 - 616) señalan hitos habitacionales o productivos en una franja homogénea al oriente de la cual se extiende el ámbito de la necrópolis (fig. 4). 


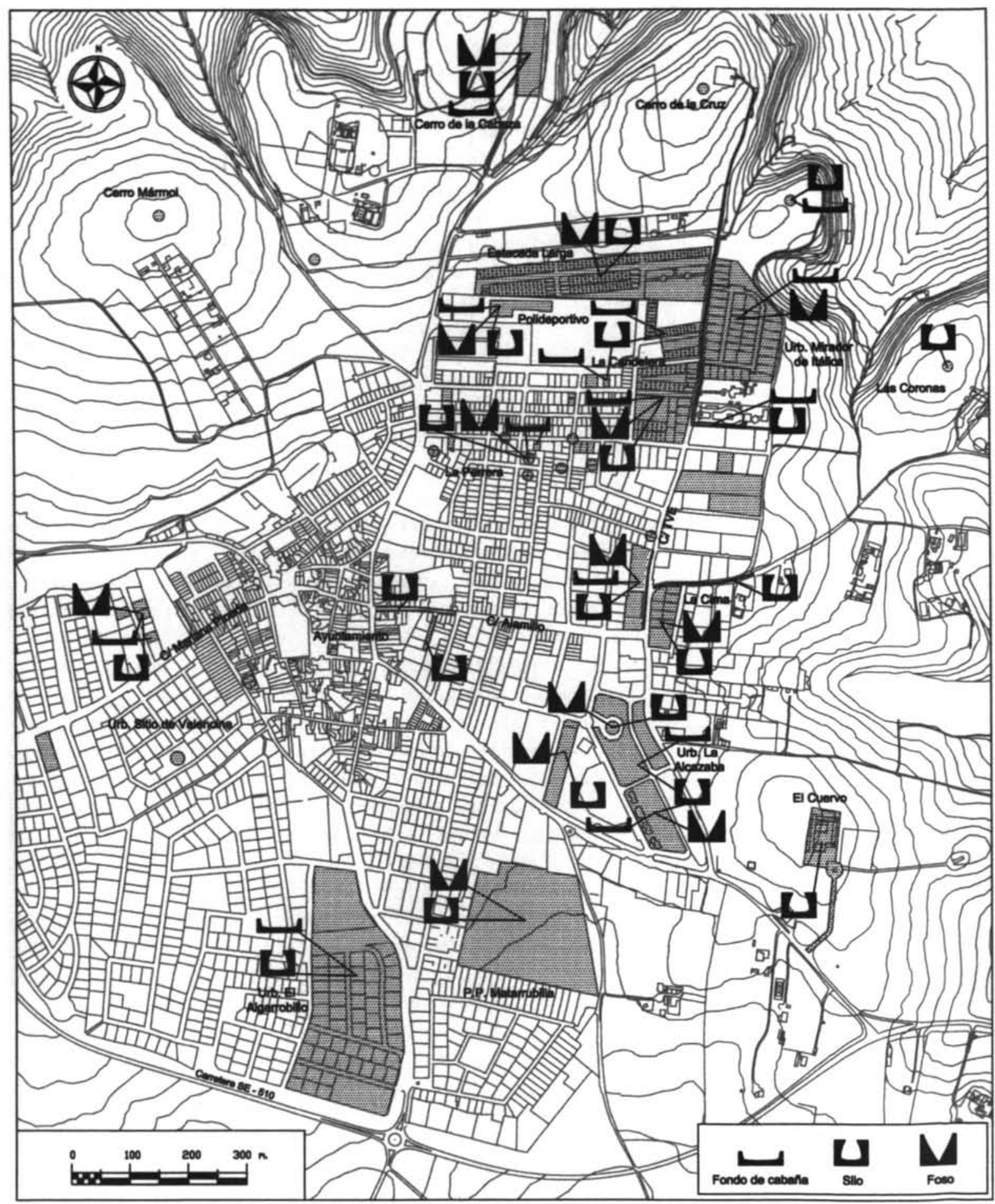

Fig. 4. Localización de estructuras del hábitat según las intervenciones arqueológicas 
De otra parte, la conjunción del análisis topográfico con las distintas evidencias arqueológicas ha mostrado su indudable valor, sobre todo si tenemos presente que hasta principios del siglo XX, la mayor parte del yacimiento se encontraba en área rústica y poseemos antiguas representaciones cartográficas en las que es posible apreciar interesantes características del relieve, antes de que su morfología fuese alterada por los procesos de mecanización del campo primero y la posterior expansión del núcleo urbano.

De este modo, en planos como el topográfico de 1906 -utilizados para las restituciones planteadas-, se dibuja claramente una gran superficie al norte y oeste de la localidad, ceñida por una curva de nivel de cota $150 \mathrm{~m}$ y situada justo al pie de la antigua carretera de Aznalcollar. Su trasposición a la realidad actual vendría a coincidir con el área en el que se localizan la mayoría de los elementos vinculables al hábitat prehistórico de Valencina, pues con los actuales levantamientos planimétricos y la restitución de primitivos relieves, se señala una extensión de las localizaciones no funerarias, en una amplia zona enmarcada por la banda situada mayoritariamente sobre la cota $150 \mathrm{~m}$-con algún registro inferior al suroeste-, siendo especialmente significativa la coincidencia de esta línea con la separación funcional que nos marcan los hallazgos orientales, como por ejemplo entre los funerarios de Mataherrera o Roquetito y los habitacionales y/o productivos de la intervención de El Cuervo.

La situación reflejada por la planimetría antigua, la realidad topográfica actual y su adecuación con las localizaciones de cabañas, fosos y silos prehistóricos, no pensamos sean elementos que casualmente coinciden.

Por otro lado, y respecto a las construcciones funerarias, un total de veinte conjuntos o localizaciones estructurales determinan la fijación de un paisaje en el que resalta la individualización de determinadas construcciones ocupando preeminencias del terreno; no solo exclusivamente al este (La Pastora, Matarrubilla, Ontiveros, Divina Pastora, Montelirio), sino también y en compañía de estructuras del hábitat, al norte (La Escalera, Cerro de la Cabeza, Cerro Mármol) y al sur (Algarrobillo, Sitio de Valencina) (fig. 5).

En el sector oriental, donde las evidencias son exclusivamente funerarias, se ha constatado una amplia ocupación, que en lo investigado, ha ofrecido profusión de estructuras de entidad menor, emplazadas en los espacios intermedios que se disponen entre las cúspides. La articulación espacial de esos hitos de mayor preeminencia, que señalan a los monumentos más relevantes, supone una clara adaptación a la morfología topográfica preexistente, en un proceso de distribución territorial en el que no se constatan distancias inferiores a los $400 \mathrm{~m}$ entre cada una de aquellas estructuras de mayor monumentalidad. Esta área, conforme a la ubicación de los hallazgos más extremos, parece tener su límite en torno a la línea sinuosa a cota $130 \mathrm{~m}$, que partiendo de los terrenos de Torrijos, se adentra en Castilleja de Guzmán, bordeando la cañada del arroyo de Montijo, para proseguir por La Escalera hasta conectar con el área del poblado en la zona de Las Coronas.

Algunos ejemplos de construcciones funerarias emplazadas en contextos espaciales de estructuras habitacionales y productivas comoel tholos del Cerro de la Cabeza (Fernández y Ruiz 1978: 193-221, Fernández y Oliva 1986: 19-33) o la cueva artificial del Algarrobillo (Santana 1993), junto a un buen número de restos óseos humanos asociados a fosos, silos o cabañas (Alcázar y otros 1992: 26), plantean la cuestión de la conjunción de ambos tipos de elementos, lo que a nuestro juicio no se debe separar del debate en torno a la sincronía de diferentes zonas, pues teniendo en cuenta el recorrido histórico del yacimiento, cabría valorar si la presencia de estos elementos, ya sean propiamente constructivos oúnicamente antropológicos, es anterior, simultanea o posterior a las evidencias habitacionales y productivas que les acompañan.

Es por todo ello de gran interés la correcta fijación de cada una de las fases del yacimiento en su asociación a los elementos arqueológicos ubicados en el espacio, sin la cual difícilmente se podrá apreciar esa relación entre ambos tipos de espacios, ni igualmente, estimar la verdadera significación de la totalidad del enclave prehistórico de Valencina que tal cual aparece valorado (por yuxtaposición de elementos) debe reflejar una realidad concerniente a varios momentos temporales. 


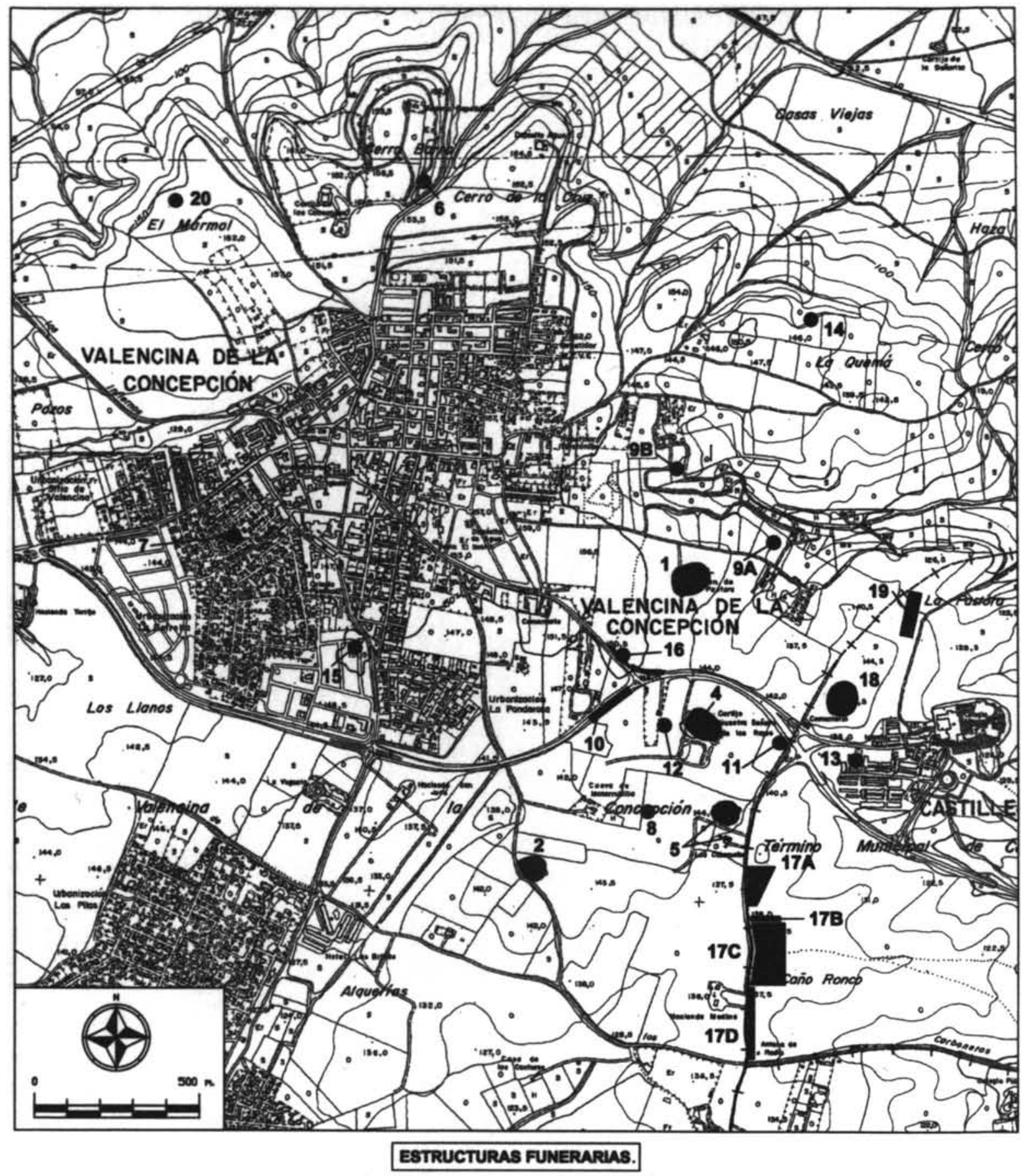

\begin{tabular}{|c|c|c|c|c|}
\hline 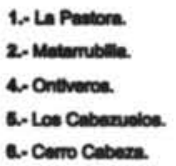 & 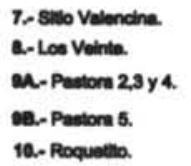 & 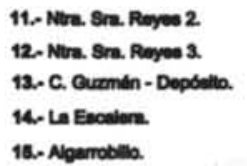 & 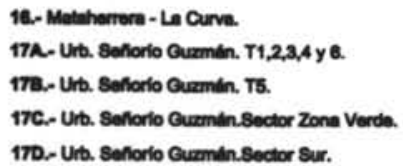 & 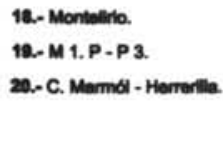 \\
\hline
\end{tabular}

Fig. 5. Localización de las estructuras funerarias prehistóricas. 
La implantación de las diferentes estructuras, no permite de momento, más que la individualización de dos grandes zonas diferenciadas por el carácter exclusivamente funerario de una de ellas. Sin embargo, la constatación en el cuadrante noreste del actual casco urbano de Valencina, de las fechas más antiguas con continuidad hasta las más recientes, así como una mayor conservación estratigráfica, superposición y concentración de estructuras arqueológicas, a la par que diversificación en los tipos, otorgan un carácter diferencial a este sector.

Al respecto, señalar por ejemplo la complejidad de los elementos arqueológicos de las excavaciones de La Perrera (Fernández y Oliva 1985, Martín de la Cruz y Miranda 1988); la yuxtaposición y densidad de estructuras en La Gallega, con 23 estructuras en $62 \mathrm{~m}^{2}$ excavados (Martín y Ruiz 1992); o en La Emisora con 38 estructuras en $272 \mathrm{~m}^{2}$ (Murillo 1991); la diversificación funcional señalada por la localización de posibles talleres en La Emisora o los hornos de La Perrera y de La Emisora; así como también la presencia de unas potencias estratigráficas sobre los limos que aunque no linealmente homogéneas, en este ámbito presentan los mayores valores con ejemplos como los más de $2.00 \mathrm{~m}$ del Corte A de La Perrera, los aproximadamente $1.50 \mathrm{~m}$ de la excavación en el Polideportivo (Murillo 1987), los 0.80/0.90 m de C/ Duero, 39 (Blanco 1991), los 0.40/0.70 m de La Emisora o los $2.00 \mathrm{~m}$ de la intervención denominada La Gallega. Frente a esta situación, la potencia en las excavaciones de la periferia es significativamente menor, con bastantes ejemplos de conservación estratigráfica prácticamente nula, en los que bajo una escasa cubierta vegetal se detectan inmediatamente los limos basales en los que se excavan el conjunto de estructuras arqueológicas. Ello además cuando las condiciones de conservación han sido semejantes, pues en todos los casos se trataba de terrenos rurales que pasan a urbanizarse e inicialmente sometidos a idénticos agentes erosivos tanto naturales (escorrentías) como antrópicos (explotación agrícola); por lo que debemos entender que una mayor conservación en el sector analizado, es seguro obedece a un grado superior de actividad antrópica que ha provocado más acumulación deposicional de carácter arqueológico.

La concurrencia de los anteriores argumentos con la morfología de la topografía antigua que hemos restituido más arriba apuntan a la diferenciación de un Área Nuclear dispuesta sobre la meseta central que englobaría los terrenos del ensanche noreste de la localidad de los años 60, 70 y 80 , los cuales en buena parte comprendían las antiguas fincas rústicas denominadas La Estacada Larga (popularmente conocida como La Perrera) y La Candelera (fig. 2).

Por lo demás, la distribución de fondos de cabañas, fosos/zanjas, silos, etc, es generalizada en la máxima superficie que alcanzó el hábitat, sin que las evidencias muestren mayores distinciones zonales que las ya mencionadas concentraciones estructurales del área nuclear, explicables por la reutilización de los espacios en diacronía, o los puntuales casos de detección de construcciones funerarias en la periferia del hábitat como en El Algarrobillo, Cerro de la Cabeza, Cerro Mármol o Sitio de Valencina, que se emplazan en elevaciones, en parte hoy desdibujadas, conforme a un esquema de implantación equiparable al reflejado por lo monumentos mas notorios del área exclusiva de necrópolis.

Las estructuras de habitación y producción se documentan por todas partes, siendo en cualquier caso general la escasez cuantitativa de las primeras, de modo que se intuye más que una concentración del hábitat, un modelo de ocupación dispersa articulado conforme a diferentes unidades básicas que englobarían funcionalidades domésticas (cabañas) y productivas (silos de almacenaje, talleres o cercados para ganado) situadas en torno a las primeras y ocupando mayores porcentajes de terreno.

De este modo se explicaría la gran extensión del área denominada habitacional y productiva, y su propia definición como tal, entendida no como una concentración de cabañas, sino como un paraje de hábitat disperso, con grandes zonas anexas de actividad agropecuaria, artesanal o de almacenamiento. En las cuantificaciones de cada uno de los tipos de estructuras arqueológicas, las de índole productiva son absolutamente mayoritarias en cualquier zona del área no funeraria. 
En este contexto, algunos de los fosos/zanjas cabría explicarlos en su adaptación a estas unidades básicas, ya sea como elementos de separación y/o compartimentación de espacios, ya como verdaderas zanjas de desagüe asociadas. Al respecto resultaría clarificador la generalización de las excavaciones en extensión y la conexión de los datos sectoriales procedentes de diferentes localizaciones, para la reconstrucción de los trazados y la posible separación de diferentes ámbitos dentro de la totalidad del área ocupacional y productiva.

De todo ello resulta nuestra hipótesis de delimitación de la totalidad del yacimiento prehistórico de Valencina, en la que de momento se han separado dos grandes ámbitos: el Área de Ocupación Habitacional y Productiva, y el Área Exclusiva de Necrópolis. La primera mayoritariamente concentrada en el actual casco urbano, engloba tanto la primitiva meseta central como los cabezos alomados de la mitad meridional así como los cerros septentrionales asomados a la cornisa y todavía en terrenos rústicos (Cerro Mármol o Goro, Cerro Barro o de las Cabezas, Cerro de la Cruz y Las Coronas). La segunda, al sureste de la primera, supera los límites administrativos de Valencina, hasta alcanzar a la localidad de Castilleja de Guzmán extendiéndose por terrenos de La Escalera, La Quemá, La Pastora, Matarrubilla, o Los Cabezuelos entre otros (fig. 6).

Del dimensionado de ambas áreas resulta una superficie de 235.60 ha. correspondientes a la zona de hábitat y/o productiva, así como de 233.20 ha. para el caso del área exclusiva de necrópolis. De este modo resulta una superficie total para el yacimiento prehistórico de Valencina estimable en unas 468.80 ha.

Esta caracterización referente a la extraordinaria extensión del enclave en relación con los parámetros de otros yacimientos contemporáneos, ejemplifica su singularidad, en un entorno donde los valores presentados son siempre significativamente inferiores, predominando los hábitats de pequeña extensión (de $1 \mathrm{a} 5 \mathrm{ha}$.), con algún ejemplo de gran superficie que en ningún caso supera las 100 ha. (García y Hurtado 1997: 139 y 140).

La monumentalidad y extensión de su necrópolis oriental; la singularidad de hallazgos como el conjunto de jabalinas de La Pastora, de origen local y concentradas en un número no equiparable en todo el Mediterráneo (Montero y Teneishvili 1996: 85); el potencial económico que se vislumbra en la cuantiosa concentración de silos, o el nivel organizativo necesario para la construcción y mantenimiento de estas y otras estructuras como los fosos; son un conjunto de elementos que nos deben hacer reflexionar sobre la entidad y complejidad del enclave de Valencina durante los tiempos prehistóricos concernientes al Cobre-Bronce.

En este contexto se afianzarían aquellas propuestas (Escacena y otros 1996: 29; García y Hurtado 1997: 146; Cruz-Auñón y Arteaga 1999: 605) que lo consideran como un verdadero centro de poder, capital o centro matriz de un entorno que al menos englobaría el Bajo Guadalquivir, entendido como centro económico e ideológico alrededor del cual se disponen una serie de comunidades periféricas que mantienen con él vínculos materiales y posiblemente lazos ideológicos. 


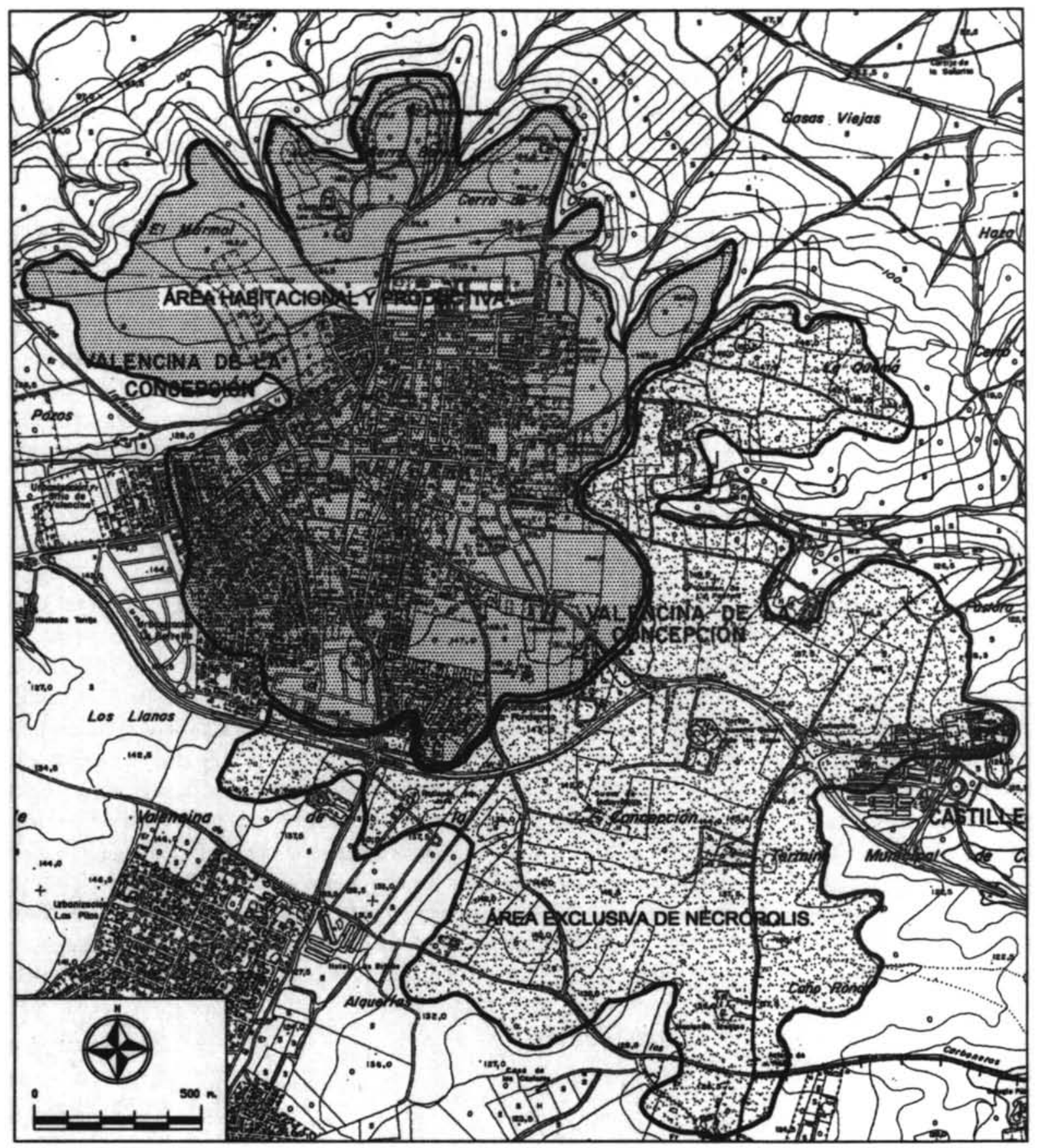

Fig. 6. Propuesta de delimitación de áreas en el yacimiento prehistórico de Valencina de la Concepción. 


\section{BIBLIOGRAFÍA}

ALCÁZAR, J. y OTROS (1992): "Enterramientos calcolíticos en zonas de hábitat", Revista de Arqueología 137: 18-27. Madrid.

ARTEAGA, O. y ROOS, A. (1995): "El proyecto geoarqueológico de las marismas del Guadalquivir. Perspectivas arqueológicas de la campaña de 1992", Anuario Arqueológico de Andalucía 1992, II :329-339. Cádiz.

ARTEAGA, O. y CRUZ-AUÑóN, R. (1999 a): "El sector funerario de "Los Cabezuelos" (Valencina de la Concepción, Sevilla). Resultados preliminares de una excavación de urgencia", Anuario Arqueológico de Andalucía 1995 III: 589-599. Sevilla.

(1999 b): "Una valoración del patrimonio histórico en el campo de silos de la finca El Cuervo-RTVA (Valencina de la Concepción, Sevilla). Excavación de urgencia de 1995", Anuario Arqueológico de Andalucía 1995 III: 608-616. Sevilla.

BAENA, R. y DÍAZ DEL OLMO, F. (1997): “Valle inferior del Guadalquivir. Excursión C", Guía de campo de la IV reunión de Cuaternario Ibérico: 65-89, Ed. Joaquín Rodríguez Vidal. Huelva.

BLANCO, A. (1991): "Excavación de urgencia en el solar de C/ Duero n³9. Valencina de la Concepción (Sevilla).", Anuario Arqueológico de Andalucía 1989 III: 430-431. Sevilla.

BELÉN, M. (2000): "El país: Territorio y poblamiento", Argantonio. Rey de Tartessos: 79-115. Sevilla.

CORTÉS, J. y OTROS (1992): Planos de Sevilla. Colección Histórica (1771-1918). Sevilla.

CRUZ-AUÑÓN, R. y ARTEAGA, O. (1999): “Acerca de un campo de silos y un foso de cierre prehistóricos ubicados en "La Estacada Larga" (Valencina de la Concepción, Sevilla). Excavación de urgencia de 1995.", Anuario Arqueológico de Andalucía 1995 III: 600-607. Sevilla.

ESCACENA, J.L.; RODRÍGUEZ DEZULOAGA, M. y LADRÓN DEGUEVARA, I. (1996): Guadalquivir salobre. Elaboración prehistórica de sal marina en las antiguas bocas del río. Confederación Hidrográfica del Guadalquivir, Sevilla.

FERNÁNDEZ, F. y RUIZ MATA, D. (1978): "El Tholos del Cerro de la Cabeza, en Valencina de la Concepción (Sevilla)", Trabajos de Prehistoria 35: 193-221. Madrid.

FERNÁNDEZ, F. y ALONSO, J. (1985): “Un fondo de cabaña campaniforme en la Universidad Laboral de Sevilla", Noticiario Arqueológico Hispánico 22: 9-26.

FERNÁNDEZ, F. y OLIVA D. (1985): "Excavaciones en el yacimiento calcolítico de Valencina de la Concepción (Sevilla). El Corte C (La Perrera)", Noticiario Arqueológico Hispánico 21: 7-131. Madrid. (1986): "Valencina de la Concepción (Sevilla). Excavaciones de urgencia", Revista de Arqueología 58: 19-33.

GALÁN, E. y PÉREZ, J.L. (1989): Geología de Sevilla y alrededores y características geotécnicas de los suelos del área urbana: 38-57. Ayuntamiento de Sevilla. Sevilla.

GARCÍA, L. y HURTADO, V. (1997): "Los inicios de la Jerarquización Social en el Suroeste de la Península Ibérica (c. 2500 - 1700 a.n.e.). Problemas conceptuales empíricos", Saguntum 30. Homenaje a la Dra. Milagros Gil-Mascarell Boscá. Vol. II La península ibérica entre el Calcolítico y la Edad del Bronce. Valencia: 139 y 140.

JIMÉNEZ, A. (1999): Informe técnico para la delimitación del yacimiento arqueológico en el parque de Miraflores (Sevilla). Sevilla. (Inédito).

MARTÍN-CONSUEGRA, E.; CHISVERT, N.; CÁCERES, L. y UBERA J.L. (1998): “Archaeological, palynological and geological contributions to landscape reconstruction in the alluvial plain of the Guadalquivir river at San Bernardo, Sevilla (Spain)", Journal of Archaeological Science 25: 521-532. 
MARTÍN DE LA CRUZ, J.M. y MIRANDA, J.M. (1988): "El poblado calcolítico de Valencina de la Concepción (Sevilla): una revisión crítica", CuPAUAM 15: 37 -67. Madrid.

MARTÍN, A. y RUIZ, T. (1992): "Excavación calcolítica de urgencia en la finca "La Gallega" 1 a Fase. Valencina de la Concepción, Sevilla", Anuario Arqueológico de Andalucía 1990 III: 455-458. Sevilla.

MONTERO, I. y TENEISHVILI, T. (1996): "Estudio actualizado de las puntas de jabalina del dolmen de La Pastora (Valencina de la Concepción, Sevilla)", Trabajos de Prehistoria 53, 1: 73-90. Madrid.

MURILLO, T. (1991): "Excavaciones de urgencia en el poblado calcolítico de Valencina de la Concepción (Sevilla), 1988-1989.”, Anuario Arqueológico de Andalucía 1989 III: 555-560. Sevilla.

MURILLO, T y OTROS (1987): "Excavación en el yacimiento calcolítico del polideportivo de Valencina de la Concepción (Sevilla), 1985.", Anuario Arqueológico de Andalucía 1985 III: 311-315. Sevilla.

PALOMO, F. (1878): Historia crítica de las riadas o grandes avenidas del Guadalquivir en Sevilla desde su reconquista hasta nuestros días. Sevilla.

ROMO, A. (1999): "El sondeo estratigráfico de la Plaza Virgen de los Reyes (Sevilla). El registro deposicional", Anuario Arqueológico de Andalucía 1994 III: 422-432. Sevilla.

RUIZ, T. (1996a): "Valencina de la Concepción: Avance de la Carta Arqueológica", Actas del XXIII Congreso Nacional de Arqueología: 37-41. Elche.

- (1996 b): Informe arqueológico sobre la excavación de urgencia en la carretera a Salteras $s / n$, junto a Pozoblanco, en Valencina de la Concepción. (Inédito).

(1999): "Excavación arqueológica de urgencia en la urbanización "El Mirador de Itálica". Valencina de la Concepción. Sevilla", Anuario Arqueológico de Andalucía 1994 III: 511-516. Sevilla.

SANTANA, I. (1993): "Excavación de urgencia en "El Algarrobillo", Valencina de la Concepción (Sevilla)", Anuario Arqueológico de Andalucía 1991 III: 548 - 553. Cádiz.

VANNEY, J.R. (1970): L'Hidrologie du bas Guadalquivir, C.S.I.C., Madrid.

VARGAS, J.M. (2004): Carta arqueológica municipal de Valencina de la Concepción. Arqueología Monografías. Ciudad y Territorio. Consejería de Cultura. Junta de Andalucía. Sevilla 